\title{
ENFERMEIRO E SUA PERCEPÇÃo SOBRE O SISTEMA MANUAL DE REGISTRO NO PRONTUÁRIO
}

\author{
Sérgio Ribeiro dos Santos ${ }^{1}$ \\ Adenylza Flávia Alves de Paula ${ }^{2}$ \\ Josilene Pereira Lima²
}

Santos SR, Paula AFA, Lima JP. O enfermeiro e sua percepção sobre o sistema manual de registro no prontuário. Rev Latinoam Enfermagem 2003 janeiro-fevereiro; 11(1):80-7.

O objetivo deste estudo foi identificar os fatores relacionados à compreensão que os enfermeiros têm frente ao sistema manual de registro de informações em enfermagem. Trata-se de um estudo exploratório-descritivo, desenvolvido com duzentos enfermeiros de instituições hospitalares da cidade de João Pessoa-Paraíba-Brasil. Os dados foram coletados por meio de questionário utilizando uma escala de atitudes, tipo Likert. Nos resultados, foram identificados cinco fatores: a) pouco conhecimento sobre sistemas de informação; b) insatisfação com o sistema manual de registro; c) necessidade de um sistema informatizado de registro do cuidado de enfermagem; d) desejo de utilizar um sistema de classificação em todas as fases do processo de enfermagem; e) dificuldades com os diagnósticos de enfermagem e o registro manual. Os resultados mostram que os enfermeiros estão insatisfeitos com o sistema de registro manual e apontam para a necessidade de mudanças no atual modelo de registro.

DESCRITORES: sistemas de informação, prontuário eletrônico, registros de enfermagem

\section{NURSES AND THEIR PERCEPTION ABOUT THE MANUAL RECORDING} SYSTEM IN PATIENTS' FILES

The main goal of this work was to identify factors related to nurses' understanding about the manual recording system, through a descriptive-exploratory approach, developed with two hundred nursing professionals working at different hospital institutions located in the city of João Pessoa/Paraíba, Brazil. Data were collected with special forms using the Likert's behavioral scale. Results identified five factors such as: a) lack of knowledge about information systems; b) dissatisfaction with the manual recording system; c) needs of an informatized recording system to be used in nursing care; d) desire to utilize a classification system with all steps of the nursing process; and e) difficulties with nursing diagnosis related to manual recording. Findings demonstrated the dissatisfaction of nursing professionals about the manual recording system, requiring deep changes in the established recording model.

DESCRIPTORS: information systems, computerized medical recording systems, nursing records

\section{EL ENFERMERO Y SU PERCEPCIÓN SOBRE EL SISTEMA MANUAL DE REGISTRO EN LA HISTORIA CLÍNICA}

El objetivo de este estudio fue identificar los factores relacionados con la comprensión que los enfermeros tienen frente al sistema manual de registro de informaciones en enfermería. Se trata de un estudio exploratorio-descriptivo, desarrollado con doscientos enfermeros de instituciones hospitalarias de la ciudad de João Pessoa-Paraíba-Brasil. Los datos fueron recolectados a través de la aplicación de un cuestionario utilizando una escala de actitudes tipo Likert. En los resultados identificamos cinco factores: a) poco conocimiento sobre sistemas de información; b) insatisfacción con el sistema manual de registro; c) necesidad de un sistema informatizado de registro del cuidado de enfermería; d) deseando utilizar un sistema de clasificación en todas las fases del proceso de enfermería; e) dificultades con los diagnósticos de enfermería y el registro manual. Los resultados demuestran que los enfermeros están insatisfechos con el sistema de registro manual y apuntan para la necesidad de cambios en el actual modelo de registro.

DESCRIPTORES: sistemas de información, sistemas de registros médicos computadorizados, registros de enfermería

\footnotetext{
${ }^{1}$ Enfermeiro, Mestre em Enfermagem, Docente do Departamento de Enfermagem Médico-Cirúrgica e Administração-DEMCA, do Centro de Ciências da Saúde, Doutorando em Ciências da Saúde, e-mail: s.r.santos@uol.com.br; ${ }^{2}$ Alunas do Curso de Graduação em Enfermagem, Bolsistas do Programa de Bolsa de Iniciação Científica - PIBIC/CNPq. Universidade Federal da Paraíba
} 
INTRODUÇÃO

Ao longo dos anos, tem predominado na prática de enfermagem, nas unidades hospitalares, o sistema manual de registro dos cuidados prestados ao paciente. Em geral, todas as informações referentes às ações e observações realizadas pela equipe de enfermagem são registradas como um meio para gerenciar a assistência e avaliar a qualidade do atendimento.

A enfermagem produz, diariamente, muitas informações inerentes ao cuidado dos pacientes. É possível estimar que ela seja responsável por mais de $50 \%$ das informações contidas no prontuário do paciente. Entretanto, compilar essa massa de informação, que cresce em progressão geométrica nos protocolos e registros manuais, torna ineficiente o gerenciamento para uma tomada de decisão racional e objetiva por parte dos enfermeiros. Além disso, há o fato de que as anotações são inconsistentes, ilegíveis e subjetivas, não havendo uma definição metodológica estruturada.

Tem-se observado que os registros no prontuário do paciente são realizados, não só pelos enfermeiros, mas também pelos auxiliares de enfermagem, por meio de observações baseadas no estado geral do paciente e nas informações médicas. Por outro lado, há perdas no registro de informações dos prontuários, na maioria dos hospitais. Some-se a isso a ocorrência de falhas nos mecanismos de armazenamento desses prontuários, gerando dificuldades quando se procura recuperar dados para fins de pesquisa e análise ${ }^{(1)}$. $O$ registro das ações de enfermagem no prontuário é um instrumento de grande significado na assistência de enfermagem, sendo indispensável para a adequada prestação do cuidado ao paciente ${ }^{(2)}$.

Analisando-se os resultados de um levantamento realizado em prontuários hospitalares, constatou-se que apenas $0,5 \%$ das anotações neles contidas eram feitas por enfermeiros. Observou-se também que as informações transcritas não revelavam qualidade, não observavam uma seqüência lógica, não objetivavam a situação do paciente, enfim não revelavam o adequado procedimento nos cuidados para atender às necessidades de cada paciente ${ }^{(3)}$.

Infelizmente, apesar de todo o avanço tecnológico, ainda existem inúmeras instituições de saúde que utilizam o método de registro manual. Observa-se, portanto, a prática de uma enfermagem que evoluiu em termos de conhecimentos técnico-científicos, mas enfrenta um grande desafio, que é aplicar a teoria na prática e adquirir novos conhecimentos a partir da experiência vivenciada pela prática (conhecimento tácito).

A compreensão do conhecimento em enfermagem é uma matéria complexa, porque os enfermeiros sabem mais do que falam e escrevem sobre sua práxis. Diante da situação descrita, a questão que norteia esse estudo é a seguinte: Qual é a compreensão que os enfermeiros têm em relação ao sistema manual de registro de informações no prontuário do paciente?

A resposta a esse questionamento possibilitou uma reflexão sobre a prática de enfermagem e o despertar para uma nova forma de registro de informações em enfermagem. Nessa perspectiva, espera-se que este estudo contribua para mostrar a necessidade de mudanças, no sentido de substituir o sistema de informação manual por um sistema mais moderno que contemple as reais necessidades do pessoal de enfermagem. Portanto, o objetivo deste estudo é identificar os fatores relacionados à compreensão que os enfermeiros têm frente ao sistema manual de registro de informações em enfermagem.

\section{PROCEDIMENTOS METODOLÓGICOS}

Tipo de pesquisa: o presente estudo, de caráter exploratório, busca proporcionar uma visão geral do fato investigado. Tem, também, um caráter descritivo, na medida em que procura descrever as características da população ${ }^{(4)}$.

Método científico: foi utilizada como método de trabalho a abordagem hipotético-dedutiva. Trata-se de uma combinação de observação cuidadosa, hábeis antecipações e intuição científica, a fim de alcançar um conjunto de postulados que governam os fenômenos a serem pesquisados. A partir daí, são deduzidas as conseqüências observáveis, verificadas por meio de experimentação e, dessa forma, refutam-se os postulados, substituindo-os, se necessário, por outros ${ }^{(4)}$.

Delimitação da pesquisa: o desenvolvimento do estudo teve como base o levantamento que foi realizado junto aos enfermeiros. A escolha foi feita por amostragem aleatória simples e de acordo com o número de 
enfermeiros e prontuários existentes no local. Nesse sentido, foram realizados cálculos de amostra para população finita, com nível de confiança de 95\% e tolerância de erro de até $3 \%$.

Local da pesquisa: o estudo foi realizado em 30 hospitais do Município de João Pessoa-PB, entre públicos, privados e filantrópicos.

Instrumentos de coleta de dados: para o levantamento dos dados, utilizou-se um questionário, composto de duas partes: a primeira contendo dados demográficos; a segunda apresentando enunciados com o objetivo de avaliar as ações práticas dos enfermeiros, seguindo um modelo similar à escala Likert, com cinco pontos de medida para as ponderações: concordo totalmente até discordo totalmente. Antes de darmos início à coleta de dados, o projeto foi encaminhado ao Comitê de Ética em Pesquisa do Hospital Universitário Lauro Wanderley, da Universidade Federal da Paraíba, processo no 35/2001, tendo sido aprovada a sua execução. Para proceder-se à coleta de dados, foram dadas algumas orientações aos respondentes para o correto preenchimento do instrumento. A seguir, em obediência às recomendações dos princípios éticos estabelecidos na Resolução no 196/96, do Conselho Nacional de Saúde ${ }^{(5)}$, os pesquisadores deixaram claro que a colaboração dos respondentes era voluntária e que estes não precisavam identificar-se, podendo o instrumento ser devolvido em branco, caso não quisessem participar do estudo.

Técnica de Análise: os dados foram analisados à luz da estatística descritiva (média, desvio padrão, variância, freqüência absoluta e relativa, etc.). A análise fatorial foi uma ferramenta valiosa que conduziu à identificação dos componentes principais de análise, fazendo-se uso da rotação do tipo varimax e eigenvalue $\geq$ 1,990 . Os itens com carga fatorial igual ou superior a 0,400 foram aceitos. O programa SPSS IPC foi usado para conduzir o processamento estatístico do estudo ${ }^{(6)}$.

\section{RESULTADOS E DISCUSSÃO}

Os questionários foram aplicados durante os meses de janeiro a maio de 2001. Os pesquisadores estiveram presentes em todas as instituições pesquisadas, numa visita preliminar, a fim de receberem o consentimento para aplicar os instrumentos com os enfermeiros. No momento da coleta de dados, não houve qualquer obstáculo para o acesso às instituições, verificando-se uma satisfatória receptividade, por parte dos gerentes de enfermagem, em acolher os pesquisadores e colaborar com o estudo.

\section{Características da população}

Participaram do estudo duzentos enfermeiros. Observando-se os dados da Tabela 1, percebe-se que a idade média da população pesquisada é de 37 anos, com maior concentração na faixa etária entre 31 e 40 anos. Por outro lado, o tempo médio de formação da população é de 10 anos e 08 meses. Essa característica referente à faixa etária dos enfermeiros do Município de João PessoaPB confirma o resultado de outro estudo em que foi verificada uma idade média de 35 anos $^{(7)}$.

Em relação ao tempo de serviço na instituição, os enfermeiros têm, em média, 7 anos e 6 meses de serviço. Significa que esses profissionais ingressam no mercado de trabalho em pleno vigor de sua produtividade técnico-científica. Ressalta-se, ainda, que 54,5\% dos enfermeiros têm apenas o curso de graduação, enquanto $36,5 \%$ têm especialização em alguma área da enfermagem. Do total de enfermeiros participantes do estudo, $69 \%$ trabalham em instituições públicas, $24 \%$, em instituições privadas, e apenas $7 \%$, em hospitais filantrópicos. Esses dados apresentaram um coeficiente de confiabilidade Alpha igual a 0,7152, mostrando que houve correlação dentro das variáveis medidas pela avaliação.

O estudo constatou também que $62,5 \%$ dos enfermeiros pesquisados são assistenciais, ou seja, estão na prática do cuidado direto ao paciente. No desempenho de suas funções, lidam com situações diversas, incluindose as dificuldades com o registro das informações manuais, necessárias a uma boa comunicação interpessoal. Essa informação é importante na prática de enfermagem, para efeito de avaliação de desempenho e da relação custo-benefício da qualidade do serviço prestado.

Essa característica assistencial também pode ser explicada pelo nível de capacitação do enfermeiro. Como se observa na Tabela 1,54,5\% dos enfermeiros pesquisados possuem apenas o curso de graduação. Portanto, esses profissionais tendem a exercer, no mercado de trabalho, atividades voltadas para a assistência de enfermagem. 
Tabela 1 - Caracterização dos enfermeiros do Município de João Pessoa-PB, 2001

\begin{tabular}{|c|c|c|c|c|c|}
\hline \multirow{2}{*}{ Variáveis } & \multirow[t]{2}{*}{$f$} & \multirow[t]{2}{*}{$\%$} & \multirow[t]{2}{*}{ Média } & \multirow[t]{2}{*}{$\begin{array}{l}\text { Desvio } \\
\text { padrão }\end{array}$} & \multirow[t]{2}{*}{ Variância } \\
\hline & & & & & \\
\hline De 21 a 30 anos & 58 & $29,0 \%$ & 37 anos & 8,62 & 74,276 \\
\hline De 31 a 40 anos & 63 & $31,5 \%$ & & & \\
\hline De 41 a 50 anos & 57 & $28,5 \%$ & & & \\
\hline De 51 a 60 anos & 11 & $5,5 \%$ & & & \\
\hline Não informaram & 11 & $5,5 \%$ & & & \\
\hline \multicolumn{6}{|l|}{ Tempo de formado: } \\
\hline De 1 a 5 anos & 61 & $30,5 \%$ & 10,7 anos & 7,10 & 50,41 \\
\hline De 6 a 10 anos & 54 & $27,0 \%$ & & & \\
\hline De 11 a 15 anos & 31 & $15,5 \%$ & & & \\
\hline De 16 a 20 anos & 30 & $15,0 \%$ & & & \\
\hline De 21 a 25 anos & 12 & $6,0 \%$ & & & \\
\hline De 26 a 30 anos & 8 & $4,0 \%$ & & & \\
\hline Não informaram & 4 & $2,0 \%$ & & & \\
\hline \multicolumn{6}{|l|}{ Tempo de serviço na instituição: } \\
\hline De 1 a 5 anos & 101 & $50,5 \%$ & 7,5 anos & 6,43 & 41,39 \\
\hline De 6 a 10 anos & 50 & $25,0 \%$ & & & \\
\hline De 11 a 15 anos & 13 & $6,5 \%$ & & & \\
\hline De 16 a 20 anos & 20 & $10,0 \%$ & & & \\
\hline De 21 a 25 anos & 12 & $6,0 \%$ & & & \\
\hline Não informaram & 4 & $2,0 \%$ & & & \\
\hline \multicolumn{6}{|l|}{ Formação acadêmica: } \\
\hline Só graduação & 109 & $54,5 \%$ & & & \\
\hline Aperfeiçoamento & 13 & $6,5 \%$ & & & \\
\hline Especialização & 73 & $36,5 \%$ & & & \\
\hline Mestrado & 5 & $2,5 \%$ & & & \\
\hline \multicolumn{6}{|l|}{ Instituição em que trabalha: } \\
\hline Pública & 138 & $69,0 \%$ & & & \\
\hline Privada & 48 & $24,0 \%$ & & & \\
\hline Filantrópica & 14 & $7,0 \%$ & & & \\
\hline
\end{tabular}

Escala de atitudes em relação ao sistema de informação manual

A escala de atitudes foi elaborada com quarenta enunciados que descreviam aspectos relacionados à prática de enfermagem na utilização do sistema de informação manual existente nas unidades de serviço hospitalares. Do total de duzentos enfermeiros, obteve-se uma variância igual a 171,94 e um desvio padrão de 13,11 para as quarenta variáveis estudadas. A média geral de intenções dentro da escala de atitude com cinco pontos ( 1 =concordo totalmente; $2=$ =concordo um pouco; $3=$ =neutro; 4=discordo um pouco; $5=$ discordo totalmente) foi de 3,37, observando-se uma variância média por itens de 1,02. 0 coeficiente de confiabilidade alpha foi igual a 0,6503 para as quarenta variáveis investigadas. Esse resultado demonstra a consistência do instrumento, porquanto todo valor para alpha maior que 0,6000 significa que houve concordância entre o instrumento e a população estudada, ou seja, existe correlação dentro das variáveis.

O teste para verificar a significância da correlação de matrizes para a análise fatorial, o teste Bartlett's de esfericidade e a medida Kaiser-Meyer-Olkin-KMO também foram identificados na amostra. Assim, o resultado encontrado na adequação da amostra pela medida KMO foi igual a 0,606. Quando a KMO se aproxima do valor 1, então há suporte para o método de análise fatorial. Por outro lado, para o teste Bartlett's de forma esferoidal, encontrou-se um valor igual a 2.313,57. Esse resultado confirma a existência de correlação entre as variáveis da escala de atitudes. Essas informações permitiram o emprego da análise dos componentes principais, calculadas por meio do programa SPSSIPC ${ }^{(6)}$.

Assim, aplicou-se o método de rotação varimax sobre as quarenta variáveis da escala de atitudes. Para maior consistência e compreensão dos resultados, somente foram considerados fatores com eigenvalue maior que 1,900. As variáveis com carga fatorial (coeficientes) acima de 0,400 foram selecionadas para efeito de análise dos componentes principais.

O processamento dos dados permitiu identificar, dentre os enfermeiros pesquisados, cinco fatores, por meio do método de rotação Varimax, com regularização Kaiser, convergindo para oito interações. De acordo com a Tabela 2 , os componentes principais receberam as seguintes denominações: fator 1 (pouco conhecimento sobre sistemas de informação): apresentou 8,92\% de variância 
e eigenvalue igual a 3,569; fator 2 (insatisfação com o sistema manual de registro): contribuiu com $8,58 \%$ de variância e eigenvalue igual a 3,433; fator 3 (necessidade de um sistema de informação de registro do cuidado de enfermagem): apresentou 7,51\% de variância e eigenvalue igual a 2,544; fator 4 (desejo de utilizar um sistema de classificação em todas as fases do processo de enfermagem): totalizou 6,36\% de variância e eigenvalue igual a 2,360; fator 5 (dificuldades com os diagnósticos de enfermagem no sistema de informação manual): apresentou 4,99\% de variância e eigenvalue igual a 1,998. Dos quarenta itens da escala de atitudes, dezesseis foram excluídos porque não apresentaram carga fatorial dentro do limite mínimo estabelecido para este estudo $(0,400)$.

Tabela 2 - Componentes principais identificados na escala de atitudes sobre sistema de informação manual entre os enfermeiros do Município de João Pessoa-PB, 2001

\begin{tabular}{|c|c|c|c|}
\hline Componentes principais & $\begin{array}{l}\text { Carga } \\
\text { Fatorial }\end{array}$ & Eigenvalues & $\begin{array}{r}\% \text { de } \\
\text { variância }\end{array}$ \\
\hline Fator 1 - Pouco conhecimento sobre sistemas de informação & & 3,569 & 8,922 \\
\hline $\begin{array}{l}\text { Sinto dificuldades em me adaptar às mudanças que ocorrem no } \\
\text { sistema de informação. }\end{array}$ & 0,735 & & \\
\hline $\begin{array}{l}\text { Escondo das pessoas a minha insegurança quanto ao uso do } \\
\text { computador na prática assistencial de enfermagem. }\end{array}$ & 0,723 & & \\
\hline $\begin{array}{l}\text { Sinto-me incapaz de usar a tecnologia da informática na minha } \\
\text { prática assistencial. }\end{array}$ & 0,719 & & \\
\hline Tenho dificuldades em dominar o conteúdo da informática. & 0,637 & & \\
\hline $\begin{array}{l}\text { Escondo das pessoas que não domino o conhecimento sobre } \\
\text { sistema de informação automatizado. }\end{array}$ & 0,614 & & \\
\hline Fator 2 - Insatisfação com o sistema manual de registro & & 3,433 & 8,582 \\
\hline $\begin{array}{l}\text { Considero o sistema manual de registro de informação limitado e } \\
\text { ultrapassado. }\end{array}$ & 0,745 & & \\
\hline $\begin{array}{l}\text { Percebo que as informações registradas pela enfermagem no } \\
\text { prontuário não são lidas pela equipe de saúde. }\end{array}$ & 0,713 & & \\
\hline $\begin{array}{l}\text { Penso que o sistema manual de registro de informação não satisfaz } \\
\text { as complexas exigências da enfermagem atual. }\end{array}$ & 0,592 & & \\
\hline $\begin{array}{l}\text { Tenho a impressão de que as informações registradas no prontuário } \\
\text { pela enfermagem não são valorizadas pelos outros profissionais. }\end{array}$ & 0,567 & & \\
\hline $\begin{array}{l}\text { Considero desmotivante fazer o registro manual dos cuidados de } \\
\text { enfermagem. }\end{array}$ & 0,550 & & \\
\hline $\begin{array}{l}\text { Penso que registrar manualmente as informações de enfermagem no } \\
\text { plano de cuidados é burocratizar o trabalho do enfermeiro. }\end{array}$ & 0,439 & & \\
\hline \multicolumn{2}{|l|}{$\begin{array}{l}\text { Fator } 3 \text { - Necessidade de um sistema informatizado de registro do cuidado } \\
\text { de enfermagem }\end{array}$} & 3,006 & 7,515 \\
\hline $\begin{array}{l}\text { Penso que, se tivéssemos um sistema de informação automatizado, } \\
\text { o enfermeiro teria mais tempo para cuidar do paciente. }\end{array}$ & 0,750 & & \\
\hline $\begin{array}{l}\text { Considero o registro do cuidado de enfermagem indispensável para } \\
\text { nortear a continuidade da assistência. }\end{array}$ & 0,739 & & \\
\hline $\begin{array}{l}\text { Penso que os problemas no registro das informações em } \\
\text { enfermagem seriam minimizados com o uso de um sistema } \\
\text { informatizado de registro. }\end{array}$ & 0,727 & & \\
\hline $\begin{array}{l}\text { Desejaria utilizar um sistema de informação em enfermagem } \\
\text { automatizado. }\end{array}$ & 0,605 & & \\
\hline \multirow{2}{*}{\multicolumn{2}{|c|}{$\begin{array}{l}\text { Fator } 4 \text { - Desejo de utilizar um sistema de classificação } \\
\text { do processo de enfermagem }\end{array}$}} & 2,544 & 6,360 \\
\hline & & & \\
\hline $\begin{array}{l}\text { Preocupo-me por não desenvolver o processo de enfermagem em } \\
\text { todas as suas fases. }\end{array}$ & 0,608 & & \\
\hline $\begin{array}{l}\text { Desejaria utilizar um sistema de classificação (diagnóstico, } \\
\text { intervenção e resultado), mas esse processo não é difundido nessa } \\
\text { instituição. }\end{array}$ & 0,567 & & \\
\hline $\begin{array}{l}\text { Sinto-me desapontado por não usar rotineiramente os diagnósticos } \\
\text { de enfermagem. }\end{array}$ & 0,480 & & \\
\hline $\begin{array}{l}\text { Percebo que os prontuários do paciente carecem de uma } \\
\text { padronização nos registros de enfermagem. }\end{array}$ & 0,450 & & \\
\hline Não consigo realizar todas as fases do pro & 0,439 & & \\
\hline
\end{tabular}


Análise dos componentes principais

Dos quarenta enunciados, apenas vinte e quatro obtiveram carga fatorial significante, ou seja, maior que 0,4000 . Dessa forma, os cinco fatores identificados receberam uma interpretação de acordo com o agrupamento desses fatores, conforme comentários a seguir:

a) Fator 1 (Pouco conhecimento sobre sistema de informação). O grupo de variáveis que compõe esse fator revela que os enfermeiros não estão familiarizados com a tecnologia da informação, nem com sistemas de informação. Essa situação tem causado insegurança quanto ao uso do computador e dificuldades no domínio do conteúdo da informática. Vale ressaltar que os enfermeiros participantes do estudo têm, em média, 10,7 anos de formados, ou seja, concluíram o curso de graduação no início da década de 1990. Convém frisar que, nessa época, os cursos de enfermagem existentes no Município de João Pessoa-PB, em geral, não possuíam, em seus currículos, disciplinas que abordassem o tema: tecnologia da informação ou sistemas de informação em enfermagem.

Atualmente, os curso de enfermagem têm, em seus currículos, disciplinas que enfocam essa temática. Portanto, espera-se que, nos próximos anos, os enfermeiros estejam mais envolvidos com esse conhecimento tão indispensável para a melhoria da qualidade e produtividade do serviço de enfermagem. Os enfermeiros precisam estar dotados de habilidades e conhecimentos necessários para atender as demandas de informação, associadas com a qualidade da assistência de enfermagem ${ }^{(1)}$.

b) Fator 2 (Insatisfação como sistema manual de registro). Esse fator revela que os enfermeiros pesquisados estão insatisfeitos com o atual sistema de registro, porque o consideram limitado e ultrapassado. Além disso, alegam que os outros profissionais não lêem o que a enfermagem faz. Assim, para os enfermeiros, o sistema manual de registro não satisfaz a complexidade da assistência de enfermagem, considerando-o desmotivante. Alegam que perdem muito tempo escrevendo e, o que é mais grave, permanecem longo tempo afastados do paciente. Vê-se, portanto, que o atual sistema contribui para aumentar a distância do enfermeiro em relação aos cuidados do paciente, permitindo, assim, que esse profissional permaneça a maior parte do tempo sentado atrás do birô.

c) Fator 3 (Necessidade de um sistema informatizado de registro do cuidado de enfermagem). Embora os enfermeiros demonstrassem pouco conhecimento e familiaridade com a tecnologia da informação, eles sentem necessidade de um sistema de informação computadorizado que permite o registro dos cuidados de enfermagem com mais rapidez e confiabilidade, a fim de que tenham condições de permanecer mais tempo com o paciente.

Naturalmente, o propósito do sistema de informação automatizado é tornar acessíveis os dados, além de garantir-lhes uma melhor organização. Mas, para isso, é indispensável que o serviço de enfermagem possua normas e rotinas de trabalho. Por outro lado, deve utilizarse de um sistema de classificação da prática (diagnóstico, intervenção, resultados) que proporcione um canal de comunicação interprofissional mais eficiente e consistente, resultando, assim, na melhoria da qualidade do cuidado de enfermagem ${ }^{(8-9)}$.

d) Fator 4 (Desejo de utilizar um sistema de classificação em todas as fases do processo de enfermagem). É interessante notar que as variáveis que formam o fator 4 estão fortemente relacionadas. A temática central é o sistema de classificação da prática de enfermagem para a realização do processo de enfermagem. Observa-se que existe entre os enfermeiros interesse em utilizar, na prática, o processo de enfermagem em todas as suas fases, desde o planejamento, inclusive com os diagnósticos de enfermagem, até a avaliação das intervenções. Assim, teriam a oportunidade de aplicar, racionalmente, a teoria na prática. Todavia, não conseguem desenvolver esse processo, por uma série de razões, destacando-se as seguintes: falta divulgação, carência de rotina para se desenvolver sistematicamente esse processo; ausência de padronização nos registos de enfermagem.

É necessário reconhecer que a utilização de sistemas de classificação, além de estabelecer padrões de cuidados, que podem ser utilizados em qualquer parte do mundo, permite uma melhoria na qualidade da assistência de enfermagem, por meio da sistematização, registro e quantificação do que enfermeiros e auxiliares/ técnicos produzem.

e) Fator 5 (Dificuldades com os diagnósticos de enfermagem e o registro manual). $\mathrm{O}$ agrupamento das 
variáveis que compõem o fator 5 revela que os enfermeiros sentem-se inseguros quanto ao uso dos diagnósticos de enfermagem. Além disso, não os utilizam na prática, porque desconhecem a sistematização e não têm tempo suficiente para elaborar o plano de cuidados. Essas variáveis apontam para o fato de que a sistematização dos cuidados de enfermagem, utilizando o sistema de diagnósticos da NANDA ${ }^{(10)}$ (North American Nursing Diagnosis Association), parece ser mais um modelo formal que não tem encontrado espaço na prática de enfermagem dos hospitais de João Pessoa-PB. Esse argumento está respaldado na visão dos enfermeiros pesquisados que demonstraram ter pouco conhecimento sobre o tema.

Pode-se inferir que a complexidade do assunto e a necessidade de mudar paradigmas na prática de enfermagem têm dificultado a implantação e a utilização dos diagnósticos de enfermagem no dia-a-dia do trabalho dos enfermeiros. Para eles, o registro torna-se algo enfadonho e desmotivante, porque, segundo esclarecem, perdem tempo fazendo registros manuais, quando poderiam estar ao lado do paciente.

Portanto, os cinco fatores ou componentes identificados retratam a necessidade de mudanças na forma de desenvolver as anotações e no processo de sistematização do cuidado de enfermagem. Espera-se que os enfermeiros busquem alternativas que facilitem seu trabalho e possibilitem maior aproximação na relação enfermeiro-paciente. Nesse aspecto, percebe-se uma preocupação em unir a humanização dos cuidados de enfermagem com os benefícios da tecnologia da informação.

A enfermagem, portanto, tem um papel importante nesse contexto, em virtude da complexidade de suas ações, bem como do volume de dados e informações que circulam em sua volta para registrar e organizar os dados clínicos no ambiente hospitalar, levando-se em consideração a diversidade de sua prática. Por essa razão, o sistema de informação em enfermagem deve ser eminentemente prático, ou seja, o seu desenvolvimento precisa ser extraído das necessidades dos profissionais de enfermagem que atuam diretamente na assistência ao paciente.

\section{CONSIDERAÇÕES FINAIS}

O sistema de informação manual, tendo em vista as observações anotadas pela enfermagem nos prontuários e outros papers, é visto pelos enfermeiros como um veículo de comunicação limitado e ultrapassado diante da moderna tecnologia digital.

Neste estudo, procurou-se identificar os fatores relacionados à compreensão que os enfermeiros têm frente ao sistema manual de registro das informações de enfermagem. Após a aplicação da análise fatorial, foram extraídos os componentes principais com base no rotação varimax, sobre as variáveis da escala de atitudes. Os resultados apontaram os cinco fatores discutidos e analisados no estudo.

Em conclusão, observa-se que os enfermeiros pesquisados encontram-se insatisfeitos com o sistema de registro manual e estão assumindo uma nova postura profissional em relação ao sistema de informação. Para reverter esse quadro, eles são favoráveis às mudanças tecnológicas que contribuam para minimizar a lacuna entre a teoria e a prática, por meio do desenvolvimento de sistemas que permitam maior envolvimento com a assistência de enfermagem e diminuam o tempo gasto com anotações.

É importante destacar que, para alcançar esse objetivo, faz-se necessária uma mudança de paradigma no desenvolvimento de sistemas de informação para uma abordagem participativa, centralizada no relacionamento social, no conhecimento explícito e tácito, na competência, na interação interpessoal e na experiência vivenciada pela prática do trabalho. Essa metodologia tem, como pressuposto básico, desenhar o sistema com o pessoal de enfermagem, buscando extrair deles a realidade de sua prática e experiência profissional.

Assim, por causa desse envolvimento no processo de construção do sistema, os enfermeiros, auxiliares e técnicos de enfermagem estão mais aptos a aceitar o sistema final, uma vez que são vistos como colaboradores ativos desse processo. Daí a necessidade de compreender e interpretar a perspectiva do usuário, para que, juntos, possam propor um novo sistema de informação que apoie as operações clínicas e o gerenciamento do serviço. 


\section{REFERÊNCIAS BIBLIOGRÁFICAS}

1. Marin HF. Informática em enfermagem. São Paulo (SP): EPU; 1995.

2. Fernandes RAQ, Salum MJL, Teixeira MB, Lemmi RCA, Miura M. Anotações de enfermagem. Rev Esc Enfermagem USP $1981 ; 15(1): 63-8$.

3. Ribeiro CM. Auditoria de serviços de enfermagem. Rev Bras Enfermagem 1972 jul/set; 25(4):91-103.

4. Gil AC. Métodos e técnicas de pesquisa social. São Paulo(SP): Atlas; 1994.

5. Ministério da Saúde (BR). Conselho Nacional de Saúde. Comissão Nacional de Ética em Pesquisa - CONEP. Resolução no 196/96. Dispõe sobre pesquisa envolvendo seres humanos. Brasília (DF): Ministério da Saúde; 1996.

6. Norusis MJ. SPSS/PC+. Professional statistics. Chicago: SPSS Inc.; 1992.

7. Santos SR, Valdevino J Neto, Costa SPR. Qualidade de vida no trabalho do enfermeiro. Revista do UNIPÊ 1998; 2(2):96-106.

8. Nóbrega MML. Equivalência semântica e análise da utilização na prática dos fenômenos de enfermagem da CIPE - versão alfa. [Tese]. São Paulo (SP): UNIFESP/EPM; 2000.

9. Goossen WTF, Epping PJMM, Abraham R. Classification systems in nursing formalizing nursing knowledge and implications for nursing information systems. Meth Inform Med 1996; 35:59-71.

10. North American Nursing Association. Diagnósticos de enfermagem da NANDA: definições e classificação - 19992000. Organizado por NANDA. Porto Alegre: Artes Médicas Sul; 2000. 